Original Research Paper

\title{
An Informetric Analysis of Studies on Influenza Vaccines and Vaccination
}

\author{
${ }^{1,2,3}$ Vadim Nikolaevich Gureyev, ${ }^{2,3}$ Nikolay Alekseevich Mazov, \\ ${ }^{1}$ Tatyana Nikolaevna Ilyicheva and ${ }^{1}$ Sergei Ivanovich Bazhan \\ ${ }^{I}$ Vector State Research Center of Virology and Biotechnology, Koltsovo, Novosibirsk Region, 630559, Russia \\ ${ }^{2}$ Trofimuk Institute of Petroleum Geology and Geophysics, Siberian Branch, \\ Russian Academy of Sciences, Novosibirsk, 630090, Russia \\ ${ }^{3}$ State Public Scientific Technological Library, Russian Academy of Sciences, Novosibirsk, 630200, Russia
}

Article history

Received: 03-09-2017

Revised: 29-11-2017

Accepted: 13-12-2017

Corresponding Author:

Vadim Nikolaevich Gureyev

Vector State Research Center of

Virology and Biotechnology,

Koltsovo, Novosibirsk Region,

630559, Russia

Tel: +7(383)335-64-47

Email: GureyevVN@ipgg.sbras.ru

\begin{abstract}
Influenza is one of highly contagious acute virus diseases that primarily affects respiratory ways. Annual seasonal epidemics afflict approximately $15 \%$ population worldwide and lead to $250,000-500,000$ deaths each year. That is why studies on influenza vaccines and vaccination that prevent spreading of the virus remain highly important. This paper presents the results of informetric analysis of publications on influenza vaccine development and vaccination against influenza based on Web of Science databases. The authors analyzed scholarly output from 2001 to 2015. We detected annual increase in the number of publications since 2002, but the highest interest to influenza vaccination was observed in 2009-2011 years due to emergence of new H1N1 virus. After 2011 we registered decrease in publication activity although this subject area is still in the focus of researchers. Journal original papers and reviews seem to be the main source of information, while a share of conference papers consistently decreased over the analyzed period. United States is a leader in the number of research with $40 \%$ of all papers which account for $55 \%$ of all citations. Elsevier's Vaccine is a primary journal publishing nearly $20 \%$ of papers; however, results of influenza vaccine development have also been published in extremely authoritative journals including The New England Journal of Medicine, Lancet, Science, British Medical Journal, Nature Medicine, etc. This also highlights the great importance of studies on the analyzed topic. Thus, the paper represents different aspects and trends in influenza vaccine development in the last 15 years using a broad range of bibliometric approaches including citation analysis, collaboration networks, revealing the most active countries studying influenza vaccine development, the most authoritative journals and funding organizations.
\end{abstract}

Keywords: Influenza Vaccine, Influenza Vaccination, Influenza Viruses, Bibliometric Analysis, Web of Science

\section{Introduction}

Influenza is highly contagious acute virus disease primarily affecting respiratory ways. It can cause diseases of different severity level varying from mild or even subclinical forms to severe form with fatal outcome. The most vulnerable so-called risk groups of population include children, elderly people and patients with chronic problems. Annual seasonal influenza epidemics may afflict up to $15 \%$ of population and can result in an estimated 250,000 to 500,000 deaths worldwide each year (WHO, 2009).

Illness is caused by influenza viruses belonged to the Orthomyxoviridae family. Their genome is represented by fragmented RNA of negative polarity. Virion outer layer includes lipid membrane with anchoraged glycoproteins Hemagglutinin (HA) and Neuraminidase (NA). HA and NA play an essential 
role in immune response against the virus, since antibodies binding to these glycoproteins protect human against infection.

Vaccination is one of the promising approach to prevent global influenza virus spreading. Vaccines against influenza $\mathrm{A}$ and $\mathrm{B}$ viruses were developed in 1940s. Those initial whole-virion vaccines were produced in embryonated chicken eggs (this technique is still predominant) and included roughly purified whole virus inactivated with formalin and phenitol (Francis et al., 1945). However, the phenomenon of antigenic drift forces researchers to revise vaccine formula each 2 or 3 years. Studies on vaccine influence on children resulted in the development of splint and subunit vaccines (Davenport et al., 1964). Currently, together with Inactivated Influenza Vaccines (IIV) public health services use Live Attenuated Influenza Vaccines (LAIV). Usually they are cold-adapted and can efficiently reproduce in upper but not in lower respiratory airwaves (Jin and Subbarao, 2014).

Despite the great number of studies devoted to the development of influenza virus vaccines, scholarly output lacks informetric analyses of publications on this significant scientific topic. Literature review enabled us to find only three bibliometric studies concerning influenza viruses (Castillo-Pérez et al., 2015; Chen et al., 2015; Hou, 2014). Interestingly, one of these papers analyzes scholarly output devoted to influenza vaccines by the example of patents (Chen et al., 2015). Therefore, our study can be of help in understanding research trends in the development of vaccines against influenza viruses in terms of bibliometric indices.

\section{Materials and Methods}

Web of Science (WoS) Core Collection bibliographic database owned by Clarivate Analytics was used for the analysis of publications (http://www.webofknowledge.com). All WoS Core Collection databases except for Emerging Sources Citation Index (ESCI) were used. ESCI accumulating data from approximately 6000 journals was added in WoS Core Collection in 2015. Thus, we decided to exclude this database since it can bias the results for recent analyzed years. When counting scholarly papers written in international collaboration, we considered papers of authors affiliated with organizations from at least two countries. When considering journal impact factor, Journal Citation Reports database (Clarivate Analytics), version of 2015, was used.

We addressed "(influenza OR flu) AND vaccin*" search terms in the searching field "Title" to cover all relevant papers on the topic of influenza vaccines and vaccination against this virus. Having access to databases since 1975, we investigated all papers from this year up to 2016 and paid special attention to the papers published in the last 15 years. Scholarly output from 2001 to 2015 was divided into three equal periods to evaluate changes in research activity and its influence in terms of citations over time. Thus, we thoroughly analyzed papers from 2001 to 2015 in terms of papers distribution according to countries, types of publications, languages, specialized research areas, citation counts, the most significant journals, funding agencies and organizations. While accounting citations, we used standard 3-year citation window, i.e., the year of publication and two following years. Data on Great Britain was combined from England, Wales, North Ireland and Scotland, divided in WoS databases. As far as China is concerned, the data was combined from People's Republic of China and Taiwan.

Most part of the study (e.g., counting total number of papers, counting citations for different periods, papers written in collaboration, number of papers funded by different agencies, etc.) was carried out using embedded Web of Science and Journal Citation Reports tools for the analysis of bibliographic and bibliometric information. Some more advanced studies were made in Microsoft Excel 2016. When processing big data packages exported from WoS, we used SciMAT open-source software tool for combining duplicate units (Cobo et al., 2012).

\section{Results}

\section{Scholarly Output on Influenza Vaccines}

From 1975 to 2015, i.e., in a 40-year period, researchers published $12,326 \mathrm{WoS}$-indexed papers on influenza vaccines, with 9655 papers $(78 \%)$ published in the last 15 years (Fig. 1). These studies are of great importance among other research on influenza with a share of almost $25 \%$. It is worth noting, that this share remains stable during the last 15 years, i.e., $25.7 \%$ in $2001-2005$; $23.17 \%$ in 2006-2010 and $25.65 \%$ in 2011-2015 which points at strong positions of this research area in overall studies on influenza.

We also analyzed correlation between the studies devoted to vaccine construction and other studies on influenza such as influenza epidemiology including molecular epidemiology, antiviral therapy, development of new antiviral drugs to prevent future pandemics (Ilyicheva et al., 2016; 2013; 2011; Svyatchenko et al., 2016). For the purpose, we analyzed overall amount of papers on influenza (Fig. 2). 


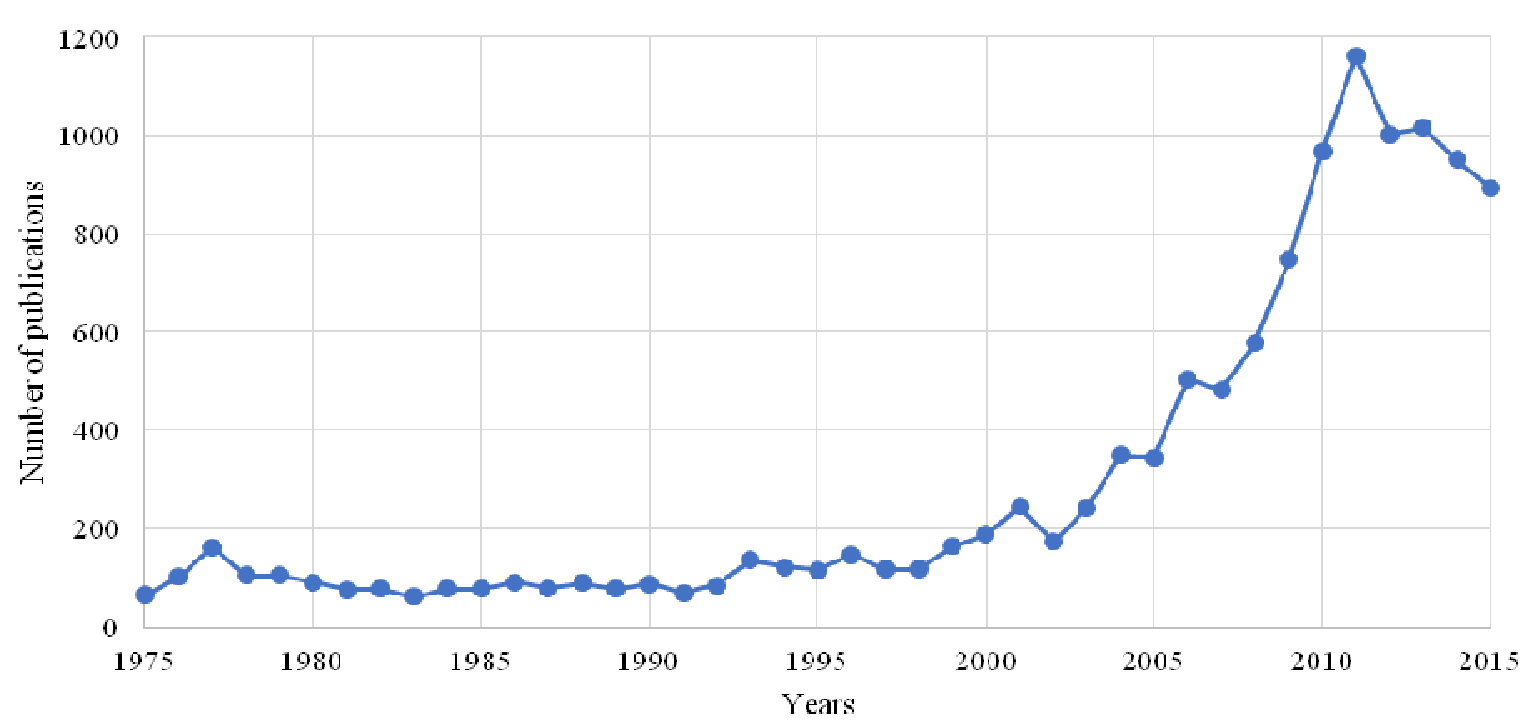

Fig. 1: Number of publications per years

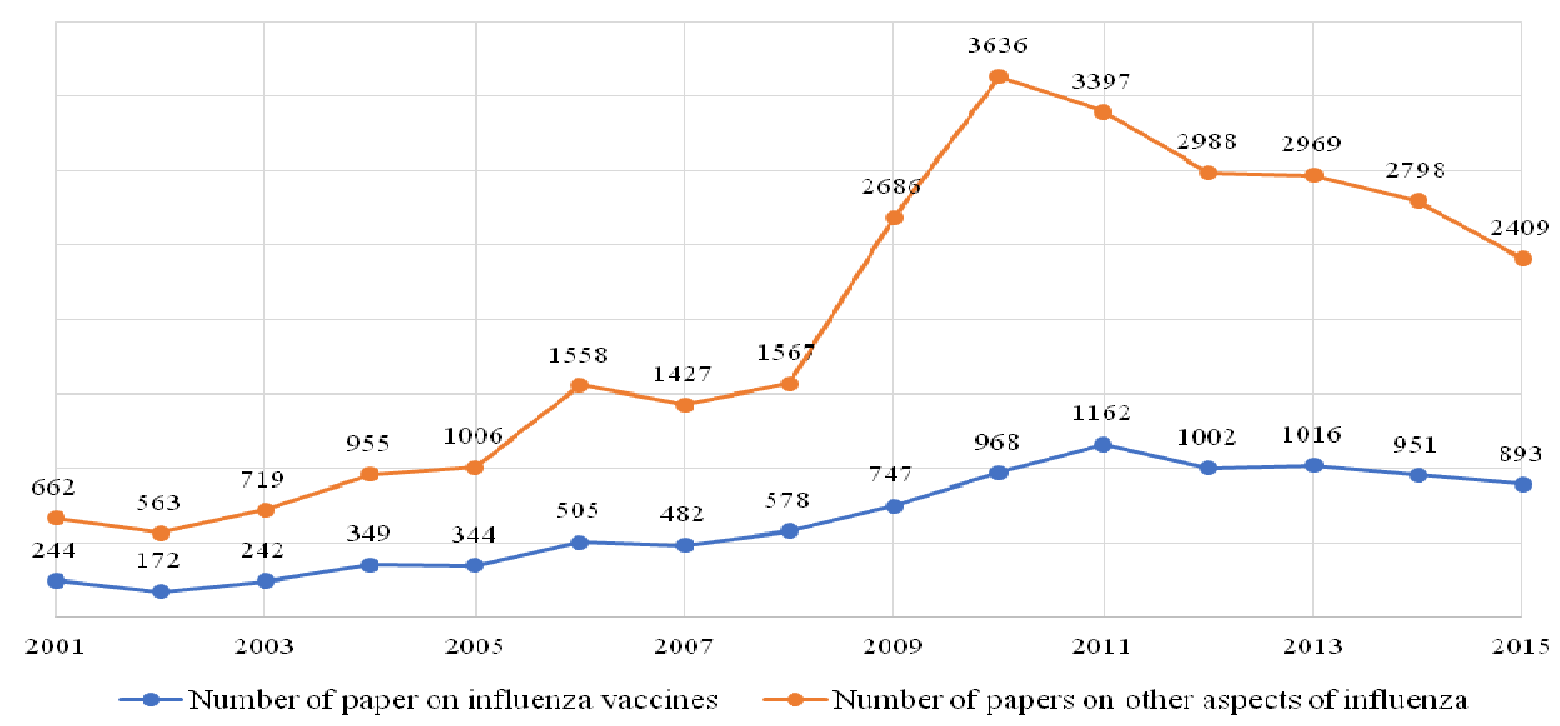

Fig. 2: Comparison of count of papers on influenza vaccines construction and papers on other influenza research

Table 1: Changes in share of different types of publications across 5-year periods. Numbers in parentheses denote a share (\%) of papers belonging to certain document type in all document collection. $\boldsymbol{\Delta}$ denotes increase in indicators; $\boldsymbol{\nabla}$ denotes decrease in indices; - means unchanged state

\begin{tabular}{|c|c|c|c|c|}
\hline \multirow[b]{2}{*}{ Document types } & \multicolumn{4}{|c|}{ Publications count and share $(\%)^{*}$} \\
\hline & 2001-2005 & $2006-2010$ & $2011-2015$ & All years $\downarrow$ \\
\hline $\boldsymbol{\Delta}$ Original articles & $731(54.11)$ & $1915(58.38)$ & $3325(66.18)$ & $5971(61.84)$ \\
\hline$\nabla$ Conference materials & $343(25.39)$ & $666(20.30)$ & $750(14.93)$ & $1759(18.22)$ \\
\hline$\nabla$ Letters & $104(7.70)$ & $228(6.95)$ & $242(4.82)$ & $574(5.95)$ \\
\hline$\nabla$ Editorials & $101(7.48)$ & $194(5.91)$ & $253(5.04)$ & $548(5.68)$ \\
\hline $\boldsymbol{\Delta}$ Reviews & $36(2.66)$ & $163(4.97)$ & $264(5.25)$ & $463(4.80)$ \\
\hline $\boldsymbol{\nabla}$ News & $89(6.59)$ & $202(6.16)$ & $169(3.36)$ & $460(4.76)$ \\
\hline - Miscellaneous & $36(2.66)$ & $89(2.71)$ & $119(2.37)$ & $242(2.51)$ \\
\hline
\end{tabular}

* In $3.6 \%$ of cases we revealed small overlapping among document types (e.g., one paper can be concurrently attributed to reviews and book chapters). Thus, total sum can be insignificantly higher than the actual number of documents 


\section{Document Types}

Table 1 shows distribution of research on influenza vaccines and vaccination across different publication types.

\section{The Most Active Countries Studying Influenza Vaccine Development}

In total, one hundred countries made contribution in engineering influenza vaccine since 2001. The first 10 out of 100 countries account for almost three-quarter (73.62\%) of all publications. Interestingly, the share of papers of all top countries among other countries have been growing steadily in all three periods and increased by $6 \%$. Distribution of scholarly output in three equal periods is demonstrated in Table 2 .

\section{The Most Influential Journals Among Scientists Studying Influenza Vaccines}

When processing journal lists, we considered the following types of documents: Articles, reviews, letters, editorials, news and errata. In total, 8124 items were published in 1161 scientific journals. Ten top journals published $36.24 \%$ papers on the analyzed topic are listed in Table 3.

\section{International Collaboration}

Table 4 demonstrates number and a share of papers published in international collaboration across 10 most productive countries. As it seen, almost in all cases except for China a share of studies conducted in international collaboration increased.

Table 2: Distribution of papers across ten most productive countries. Numbers in parentheses denote a share (\%) of papers in all document collection. $\boldsymbol{\Delta}$ denotes increase in indicators; $\boldsymbol{\nabla}$ denotes decrease in indices; - means unchanged state

\begin{tabular}{|c|c|c|c|c|}
\hline \multirow{2}{*}{$\begin{array}{l}\text { Countries with the } \\
\text { highest scholarly output }\end{array}$} & \multicolumn{4}{|c|}{ Number of publications and a share (\%) in total amount of papers } \\
\hline & 2001-2005 & $2006-2010$ & $2011-2015$ & All years $\downarrow$ \\
\hline - United States & $550(40.71)$ & $1330(40.55)$ & $2024(40.29)$ & $3904(40.44)$ \\
\hline $\boldsymbol{\nabla}$ Great Britain & $130(9.62)$ & $244(7.43)$ & $392(7.80)$ & $766(7.93)$ \\
\hline$\Delta$ Canada & $53(3.92)$ & $142(4.33)$ & $336(5.50)$ & $531(5.50)$ \\
\hline$\Delta$ China & $22(1.63)$ & $158(4.82)$ & $349(6.95)$ & $529(5.47)$ \\
\hline - Italy & $78(5.77)$ & $177(5.40)$ & $245(5.18)$ & $500(5.18)$ \\
\hline$\Delta$ France & $44(3.26)$ & $180(5.49)$ & 258 (4.99) & $482(4.99)$ \\
\hline $\boldsymbol{\nabla}$ Netherlands & $70(5.18)$ & $189(5.76)$ & $215(4.91)$ & $474(4.91)$ \\
\hline A Australia & $31(2.29)$ & $117(3.57)$ & $262(4.25)$ & $410(4.25)$ \\
\hline$\Delta$ Germany & $29(2.15)$ & $145(4.42)$ & $233(4.22)$ & $407(4.22)$ \\
\hline $\bar{\nabla}$ Japan & $65(4.81)$ & $124(3.78)$ & $210(4.13)$ & $399(4.13)$ \\
\hline
\end{tabular}

Table 3: Journals published the majority of papers on influenza vaccines

\begin{tabular}{lll}
\hline Journal & Publisher & Number and a share (\%) of papers $\downarrow$ \\
\hline Vaccine & Elsevier & $1488(18.32)$ \\
PLoS One & PLoS & $287(3.53)$ \\
Human Vaccines \& Immunotherapeutics & Taylor and Francis & $214(2.63)$ \\
Journal of Infectious Diseases & Oxford University Press & $174(2.14)$ \\
Expert Review of Vaccines & Taylor and Francis & $174(2.14)$ \\
Clinical Infectious Diseases & Oxford University Press & $141(1.74)$ \\
Influenza and Other Respiratory Viruses & Wiley-Blackwell & $140(1.72)$ \\
Journal of Virology & American Society for Microbiology & $131(1.61)$ \\
Infection Control and Hospital Epidemiology & Cambridge University Press & $104(1.28)$ \\
Pediatric Infectious Disease Journal & Lippincott Williams \& Wilkins & $91(1.12)$ \\
\hline
\end{tabular}

Table 4: Collaboration between ten countries with the largest amount of studies on influenza vaccine development and vaccination against influenza. $\boldsymbol{\Delta}$ denotes increase in indicators; $\boldsymbol{\nabla}$ denotes decrease in indices; - means unchanged state

\begin{tabular}{|c|c|c|c|c|c|c|}
\hline \multirow[b]{2}{*}{ Top countries } & \multicolumn{4}{|c|}{$\begin{array}{l}\text { Number and a share }(\%) \text { of papers published in } \\
\text { collaboration with other top countries }\end{array}$} & \multirow{2}{*}{$\begin{array}{l}\text { Number of } \\
\text { collaborating } \\
\text { countries } \\
\text { All years }\end{array}$} & \multirow{2}{*}{$\begin{array}{l}\text { Number and a share }(\%) \\
\text { of papers without } \\
\text { international collaboration } \\
\text { All years }\end{array}$} \\
\hline & $2001-2005$ & $2006-2010$ & $2011-2015$ & All years $\downarrow$ & & \\
\hline $\boldsymbol{\Delta}$ Great Britain & $37(31.36)$ & $78(34.82)$ & $164(40.07)$ & $279(39.97)$ & 61 & $383(54.87)$ \\
\hline $\boldsymbol{\Delta}$ Germany & $7(24.14)$ & $50(34.48)$ & $92(39.48)$ & 149 (36.61) & 59 & $211(51.84)$ \\
\hline $\boldsymbol{\Delta}$ Canada & $15(28.30)$ & $52(36.62)$ & $123(36.61)$ & $190(35.78)$ & 31 & $325(61.21)$ \\
\hline A Italy & $20(25.64)$ & $61(34.46)$ & $94(38.38)$ & $175(35.00)$ & 56 & $293(58.60)$ \\
\hline $\boldsymbol{\Delta}$ Netherlands & $11(15.71)$ & $57(30.16)$ & 95 (44.19) & $163(34.39)$ & 51 & $274(57.81)$ \\
\hline$\Delta$ France & $7(15.91)$ & $60(33.33)$ & $89(34.49)$ & $156(32.37)$ & 68 & $253(52.49)$ \\
\hline - Australia & $7(22.58)$ & $33(28.21)$ & $74(28.24)$ & $114(27.80)$ & 55 & $277(67.56)$ \\
\hline$\nabla$ China & $5(35.71)$ & $34(26.98)$ & $74(27.51)$ & $113(27.63)$ & 55 & $286(69.93)$ \\
\hline $\boldsymbol{\Delta}$ United States & $50(10.00)$ & $170(12.78)$ & $336(16.60)$ & $556(14.24)$ & 84 & $3062(78.43)$ \\
\hline - Japan & $16(24.62)$ & $32(25.81)$ & $52(24.76)$ & $100(25.06)$ & 47 & $281(70.43)$ \\
\hline
\end{tabular}




\section{Citation Count}

To reveal the influence of papers devoting to influenza vaccine development across countries, we analyzed citations of papers in three studied periods using 3-year citation window, i.e., the year of publication and two following years. The results of citation analysis are demonstrated in Table 5. As it seen from Table 5 and Fig. 3, research on influenza vaccination and vaccine design are primarily conducted in the United States. Furthermore, publications from the United States are the most influential in terms of number of citations. Significant progress is observed in Canada, Australia, China and
Germany. Besides, the latter country was absent among leaders in the first analyzed period. At the same time, decrease in research activity is registered in Japan, although this country is still one of the most productive. Additionally, only in Japan we registered excess of share of papers over a share of citations.

Figure 3 depicts the relation between share of papers and share of their influence in terms of citations in ten top countries.

Another popular bibliometric index characterizing influence of research papers includes number of citations per paper (Table 6).

Table 5: Citation count and a share of citations. $\boldsymbol{\Delta}$ denotes increase in indicators; $\boldsymbol{\nabla}$ denotes decrease in indices; - means unchanged state

\begin{tabular}{|c|c|c|c|c|}
\hline \multirow[b]{2}{*}{ Countries } & \multicolumn{4}{|c|}{ Citation count and a share of citations (\%) } \\
\hline & $2001-2005$ & 2006-2010 & $2011-2015^{*}$ & All years $\downarrow$ \\
\hline - United States & $3538(59.67)$ & $11519(51.75)$ & $15058(55.09)$ & $30115(54.24)$ \\
\hline $\boldsymbol{\nabla}$ Great Britain & $639(10.77)$ & $2007(9.02)$ & $2506(9.17)$ & $5152(9.28)$ \\
\hline $\boldsymbol{\nabla}$ Italy & $497(8.38)$ & $1754(7.88)$ & $1752(6.41)$ & $4003(7.21)$ \\
\hline$\Delta$ Canada & $196(3.30)$ & $1129(5.07)$ & $2124(7.77)$ & 3449 (6.21) \\
\hline $\boldsymbol{\nabla}$ Netherlands & $394(6.64)$ & $1512(6.79)$ & $1438(5.26)$ & $3344(6.02)$ \\
\hline$\Delta$ China & $117(1.97)$ & $1415(6.36)$ & $1687(6.17)$ & $3219(5.80)$ \\
\hline$\Delta$ France & $236(3.98)$ & $1443(6.48)$ & $1514(5.54)$ & $3193(5.75)$ \\
\hline$\Delta$ Germany & $119(2.00)$ & $1331(5.98)$ & $1531(5.60)$ & $2981(5.37)$ \\
\hline A Australia & $88(1.48)$ & $1262(5.67)$ & $1523(5.57)$ & $2873(5.17)$ \\
\hline $\boldsymbol{\nabla}$ Japan & $299(5.04)$ & $770(3.46)$ & $791(2.89)$ & $1860(3.35)$ \\
\hline All countries & $5929(100)$ & $22258(100)$ & $27335(100)$ & $55522(100)$ \\
\hline
\end{tabular}

*Data in the third period is incomplete since number of citations in 3-years citation window for papers published in 2015 will have increased till the end of 2017

Table 6: Citations per paper across countries during three analyzed periods. $\mathbf{\Delta}$ denotes increase in indicators;

\begin{tabular}{|c|c|c|c|c|c|}
\hline \multirow[b]{2}{*}{ Countries } & \multicolumn{4}{|c|}{ Average citations per paper } & \multirow{2}{*}{$\begin{array}{l}\text { Number and a share } \\
(\%) \text { of uncited papers } \\
\text { All years }\end{array}$} \\
\hline & $2001-2005$ & $2006-2010$ & 2011-2015 & All years $\downarrow$ & \\
\hline$\overline{\mathbf{\Delta} \text { Italy }}$ & 6.37 & 9.91 & 7.15 & 8.01 & $81(16.20)$ \\
\hline$\Delta$ United States & 6.43 & 8.66 & 7.44 & 7.71 & $830(21.26)$ \\
\hline$\Delta$ Germany & 4.10 & 9.18 & 6.57 & 7.32 & $70(17.20)$ \\
\hline $\bar{\Delta}$ Netherlands & 5.63 & 8.00 & 6.69 & 7.05 & $75(15.82)$ \\
\hline$\Delta$ Australia & 2.84 & 10.79 & 5.81 & 7.01 & $67(16.34)$ \\
\hline $\boldsymbol{\Delta}$ Great Britain & 4.92 & 8.23 & 6.39 & 6.73 & $194(25.33)$ \\
\hline $\bar{\Delta}$ France & 5.36 & 8.02 & 5.87 & 6.62 & $104(21.58)$ \\
\hline A Canada & 3.70 & 7.95 & 6.32 & 6.50 & $124(23.35)$ \\
\hline $\boldsymbol{\nabla}$ China & 5.32 & 8.96 & 4.83 & 6.09 & 74 (13.99) \\
\hline $\boldsymbol{\nabla}$ Japan & 4.60 & 6.21 & 3.77 & 4.66 & $59(14.79)$ \\
\hline All countries & 4.39 & 6.79 & 5.44 & 5.75 & $2591(26.84)$ \\
\hline
\end{tabular}

Table 7: Distribution of citations of papers on influenza vaccination and vaccine development. Numbers in parentheses denote a share $(\%)$ of papers in all document collection

\begin{tabular}{lll}
\hline Distribution of citations & Publications 2001-2015 & Total number of citations (2001-2015) \\
\hline 0 & $2591(26.84)$ & 0 \\
$1-10$ & $3600(37.29)$ & $15923(10.57)$ \\
$11-20$ & $1382(14.31)$ & $20516(13.61)$ \\
$21-30$ & $702(7.27)$ & $17533(11.63)$ \\
$31-40$ & $409(4.24)$ & $14311(9.50)$ \\
$41-50$ & $252(2.61)$ & $11406(7.57)$ \\
$51-100$ & $498(5.16)$ & $34312(22.77)$ \\
$101-200$ & $180(1.86)$ & $24242(16.09)$ \\
$201-639$ & $41(0.42)$ & $12451(8.26)$ \\
Total: & $9655(100)$ & $150694(100)$ \\
\hline
\end{tabular}




\section{Highly Cited Papers}

Table 7 indicates distribution of citations across the analyzed period. According to recommendations described in (Ivanov et al., 2014), papers with number of citations higher than 30 can be considered as highly cited. Thus, 1380 papers $(14.29 \%)$ on influenza vaccination and vaccine engineering acquire high amount of citations.

Heavily cited documents include 41 papers with number of citations higher than 200. They are published in 17 journals mainly with high Impact Factors (IF) including the following:

New England Journal of Medicine (NEJM) (IF 72.406) 11

Vaccine (IF 3.235)

Lancet (IF 47.831)

Lancet Infectious Diseases (IF 19.864)

Journal of Virology (IF 4.663)

Archives of Internal Medicine (IF 17.333)

British Medical Journal (IF 20.785)

International Journal of Epidemiology (IF 7.738)

Journal of Clinical Investigation (IF 12.784)

Journal of Immunology (IF 4.856)

Journal of Infectious Diseases (IF 6.273)

Mbio (IF 6.956)

Nature Immunology (IF 21.506)

Nature Medicine (IF 29.886)

Proceedings of the National Academy of Sciences

of the USA (PNAS) (IF 9.661)

Scandinavian Journal of Immunology (IF 2.256)

Science (IF 37.205)

Only three journals highlighted in bold make the list of serials published the majority of papers on influenza vaccination and vaccine development (Table 3).

\section{Financing of Studies on Influenza Vaccination and Vaccine Development}

Research on vaccines and vaccination comprise both basic and applied approaches and, thus, require large investments. In this section, we analyze financing organizations supporting studies on influenza vaccination. More than one third of studies (3286 papers, i.e., $34 \%$ ) were financially supported and indicated financing in acknowledgement sections. The most important financing organizations include the following:

- National Institutes of Health (NIH) of the United States of America acquiring financing from the Department of Health and Human Services. NIH were acknowledged in 1142 publications (more than $35 \%$ studies with financial information in acknowledgement sections). A half of NIH financial information (568 studies) is accounted for by National Institute for Allergy and Infectious
Diseases (NIAID) which is a part of NIH. Vaccine Research Center belonging to NIAID is of particular importance in studies on influenza vaccination and vaccine development

- Centers for Disease Control and Prevention (CDC) which were mentioned in 329 papers $(10 \%)$

- GlaxoSmithKline pharmaceutical company - 261 (approximately 8\%) supported studies on influenza vaccines

- Sanofi Pasteur which is the vaccines division Sanofi pharmaceutical company. Sanofi grants are indicated in 245 (7.5\%) papers

- MedImmune which is subsidiary of AstraZeneca pharmaceutical company provided financial support for 174 research $(5.3 \%)$

- Novartis International AG pharmaceutical company was acknowledged in 154 (4.7\%) papers

As it seen, multinational pharmaceutical corporations together with NIH and CDC play significant role in enhancing and supporting scientific research on influenza vaccines. Among other large sponsoring organizations researchers mentioned Pfizer Inc. (and purchased Wyeth company), Merck \& Co., Roche Holding AG, etc. Except for NIH and CDC with headquarters located in the United States, public health organizations in other countries play less important role as compared to top pharma companies.

\section{Discussion}

Since early 2000s significant increase in studies on influenza vaccine development is observed (Fig. 1), whereas research in previous years were conducted without sharp outbreaks. The most rapid enhancement of studies is registered on the cusp of the 1st decade of the current century when the 2009 H1N1 influenza pandemic boosted the number of works devoted to the design of a vaccine against influenza. Although morbidity and mortality were not extremely high, it is new virus itself and its influence on human that may explain the phenomenon of heightened interest of researchers. Scientists and health care workers have also paid significant attention to the effectiveness of vaccines developed using various approaches. Since prevaccinal immunity was almost absent, researchers could evaluate vaccine immunogenicity and protectivity more accurately. Of note, trends in the number of patents on influenza vaccines is very close to trends in the number of research publications (cf. Fig. 2 in (Chen et al., 2015) and Fig. 1 in current paper).

Figure 2 depicts the place of studies on influenza vaccine development among other studies devoted to different aspects of influenza spreading, its functioning, prevention, etc. In general, there were no any sharp fluctuations and a share of studies on influenza vaccine 
construction correlates with a share of other influenza research. However, other studies on influenza seem to be more sensitive to the external factors such as pandemic of 2009 when amount of studies sharply increased by $72 \%$. As for studies on vaccine construction, their basic character stipulates more smooth distribution of papers per years. Nevertheless, we should note that 2009 pandemic induced researchers to conduct more studies on vaccine construction and a share of newly published papers on this topic exceeded a share of other influenza studies since 2011. When comparing average increase in a number of papers in three periods, we observed 3.7fold increase both in studies on vaccine development and other influenza studies. In total, approximately twenty influenza vaccines have been successfully developed in the analyzed period (Krammer and Palese, 2015).

In Table 1 we show distribution of publications on influenza vaccine development across various document types. It was revealed that serials publishing articles, reviews, editorials, letters and news remain the most important source for information distribution among scientists working on influenza vaccine development. We detected increasing role of serials across three analyzed periods, while a share of other types of publications have been steadily decreased. Thus, despite the overall increase in the number of publications, written communication is currently believed to be more preferred for information dissemination as compared to oral one, since a share of reports at conferences have declined.

Analysis of distribution of papers across top countries (Table 2) shows that studies on influenza vaccine development are primarily conducted in North America and Europe. Interestingly, the list of top countries remains virtually the same across years and distribution of a share of papers seems to be stable over all analyzed periods. We registered an increase in the amount of scientific production only in China, Germany and Australia. It is worth to note that China and Germany were absent in the top list in the first period, but in 2000 s they succeeded to take Spain's and Switzerland's places. Our data on top countries developing influenza vaccines are consistent with that obtained by Chen et al. (2015) analyzed patent output on influenza vaccine development.

As for representation of papers on influenza vaccine development in scholarly journals (Table 3), the obtained results demonstrate that journals publishing studies on influenza vaccine development belong mainly to large publishing companies and universities. Analysis of distribution of papers across the journals strictly confirmed Bradford's law (Bradford, 1950), since the first $20 \%$ of journals (232 items) accumulated $80.2 \%$ of papers devoted to influenza vaccine design and vaccination against influenza. Top list of journals underwent insignificant changes across three analyzed periods. However, we observed growing variability of journals over the time: in the first period results of the studies were published in 314 journals, in the middle period - in 597 serials and in the last period - in 848 journals. Wider set of journals may imply the growth of cross-disciplinary research and using the progress of other neighboring disciplines. Of note, the role of Elsevier's journal Vaccine seems to be unique in analyzed subject area, since it accumulates almost one fifth of all papers on the topic. We previously analyzed other subject areas using bibliometric approaches and share of papers in top journals were significantly lower (see, e.g. (Ilyichev et al., 2016; Mazov et al., 2017)).

Our analyses (Table 4) revealed that studies on influenza vaccine development tend to include more countries and become more international. However, there are some differences in each case. For instance, although a share of collaboration enhanced in the United States, this country has the lowest dependence on other countries and the highest share of papers written without collaboration with other countries. A different situation arises with European countries, where a share of collaborative papers increased two-fold since the early 2000s. Studies in Australia, China and Japan seems to have local character showing the highest share of internal investigations and low share of collaboration, like the USA. In case of China and Japan it can be explained by a language barrier. Besides, all three countries have long geographic distance from each other and European countries which in their turn have the highest level of collaboration.

Data in Fig. 3 and Table 5 and 6 demonstrate our results concerning citation count and uncited papers. In general, data from Table 6 depicting data on average citations per paper and a share of uncited papers confirm that of Table 5 showing data on citation count. Interestingly, in all cases regarding the number and share of publications, number and share of citations and citations per paper we observed considerable increase during the second period, primarily due to the influenza pandemic of 2009. More detailed data per years (not published here) point at the most intensive research activity in 2009-2010 years. Since 2011 or 2012 in different countries we registered sharp decrease both in the number of publications and citations. However, overall level is higher in the end of the third analyzed period as compared to the first one. In the second period of the most intensive research on influenza vaccination and vaccine design the most influential papers were published by Australian, Italian and German researchers according to data on citations per paper. 


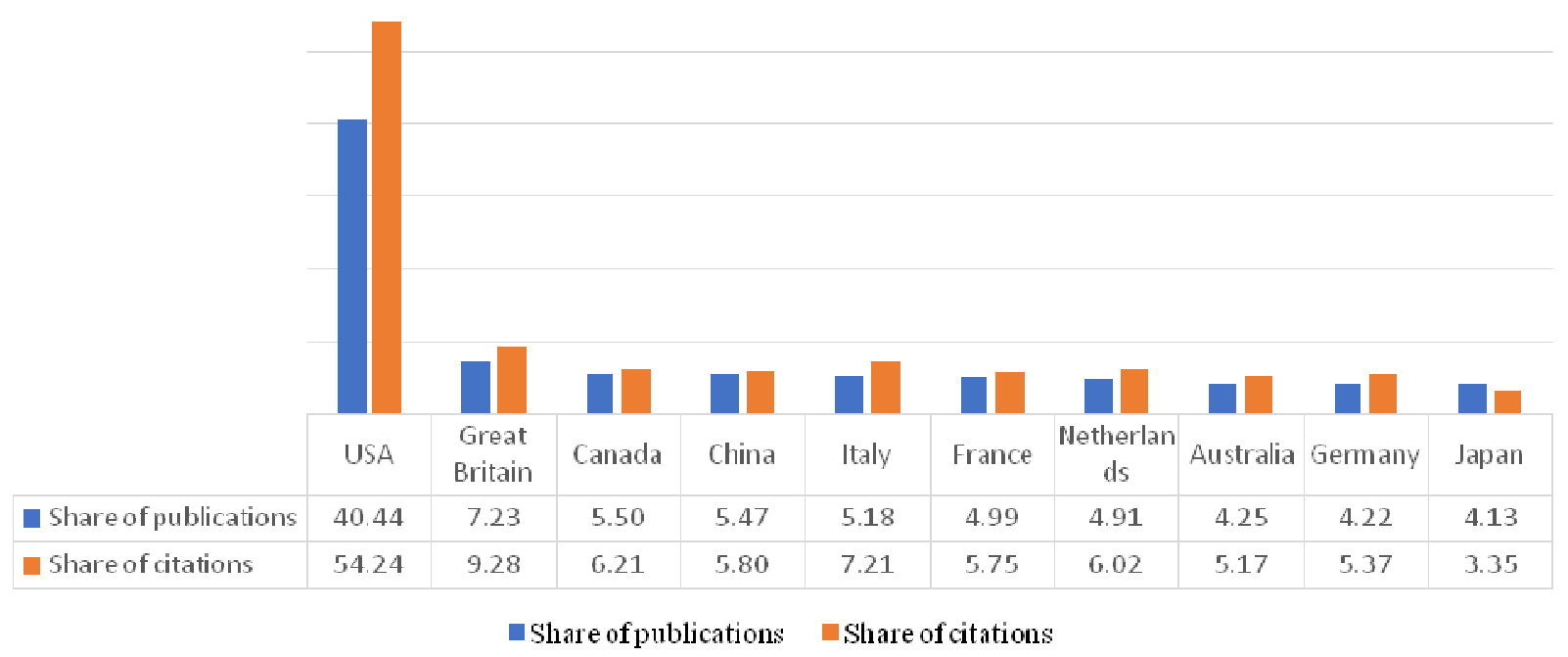

Fig. 3: The relation between a share of papers and citations across the most productive countries

Unexpected results were obtained when analyzing uncited papers. The lowest number of uncited papers belong to China and Japan which points at stable interest to Chinese and Japanese research on influenza vaccination and vaccine development. In contrast, both these countries show the lowest level of average citations per paper. As far as countries with the highest amount of uncited papers are concerned (e.g., Great Britain, France or USA), they have rather high level of average citations per paper. We may assume that the level of Chinese and Japanese studies on analyzed topic is solid and average, i.e., there are no large gap between outstanding and low-quality publications. As for Great Britain, France and USA, the difference between highly cited and uncited papers are larger. More detailed analyses can explain this phenomenon.

Analysis of highly cited papers revealed that 1380 papers acquired more than 30 citations which implies significant importance of the development of this research area. 41 papers were heavily cited and acquired more than 200 citations. Interestingly that only 7 of highly cited papers were reviews and meta-analyses which are typically acquire the majority of citations as compared to other document types (Colledge and Verlinde, 2014). All other publications belong to original research paper document type.

Generally, these papers discuss efficiency of licensed vaccines. Researchers acknowledge that all used vaccines effectively induce response of immune system in children, young and middle-aged people. However, they fail to induce high response in people older than 65 ; therefore, additional studies on development of newgeneration vaccines are necessary. Researchers introduced changes in formulas of modern vaccines to stimulate higher protection against seasonal influenza virus strains. Several new formulas (approximately 20 vaccines) were examined to induce stronger, broader and more stable immune response, especially in elderly people. There is a broad range of new formulas varying from vaccines with high dosage for elderly adults to a number of vaccines with adjuvants. Two most significant formulas MF56 and AS03 were compared with vaccines against seasonal influenza virus and revealed higher efficiency.

As is often the case, high-impact research on analyzed topic were published in multidisciplinary authoritative journals including PNAS and Science or journals of general medical nature. It is notable that only 12 of 41 highly cited papers which is less than one third were published in international collaboration. Despite registered increase in the number of collaborative research (Table 4) in the majority of countries, individual research teams still maintain paramount positions in the development of vaccines and vaccination against influenza.

Evaluation of number of financing organizations supporting studies on influenza vaccine development revealed that more than one third research were sponsored by the largest international pharmaceutical companies, NIH and CDC. Intense interest and large investments of those companies and organizations into prevention of influenza spreading using vaccination as a leading approach demonstrate high topicality and importance of vaccine engineering approaches. Vaccination is believed to be a leading approach as opposed to others. Thus, etiotropic anti-influenza drugs, especially antineuraminidase drugs, are recommended to be used together with vaccination. Broad spectrum antiviral drugs such as interferon or dugs inducing interferon production are used significantly more rarely. 


\section{Conclusion}

Results obtained in this study demonstrate significant value of approaches based on influenza vaccine development when controlling and preventing influenza spreading: A quarter of all influenza studies accrued to vaccine development approaches. Interestingly that interest of researchers and pharmaceutical companies to studying the virus depends on influenza epidemics and pandemics, as confirmed by significant increase in the number of papers and citations after H1N1 pandemic of 2009 and the subsequent decrease. Papers on influenza vaccines have been regularly published in the most authoritative journals including SpringerNature journals, NEJM, Lancet, PNAS or Science. The United States is the most productive country with the highest number of research on the topic. However, Germany, Australia and China have considerably progressed in technologies of influenza vaccines in the last 15 years. Heightened interest to vaccine development is also confirmed by participation of the largest international pharmaceutical companies in supporting such studies.

\section{Acknowledgement}

The authors are grateful to reviewers for their valuable comments that helped us to improve this paper.

\section{Funding Information}

This work was supported by Grant \#15-15-00047, Russian Science Foundation, http://rscf.ru.

\section{Author's Contributions}

All authors have read and approved the final version on the manuscript.

Vadim Nikolaevich Gureyev: Collected and processed bibliometric data, structured and planned the study and wrote the manuscript.

Nikolay Alekseevich Mazov: Designed bibliometric study.

Tatyana Nikolaevna Ilyicheva and Sergei Ivanovich Bazhan: Analyzed and discussed obtained findings, wrote the manuscript.

\section{Ethics}

This article contains unpublished material. The corresponding author confirms that there are no ethical issues concerning this study.

\section{References}

Bradford, S.G., 1950. Documentation. 1st Edn., Public Affairs Press, Washington, D.C., pp: 156.
Castillo-Pérez, J.J., L. Muñoz-Valera, F. García-Gómez and J.M. Mejía-Aranguré, 2015. Bibliometric analysis of scientific output on influenza in Mexico, 2000-2012. Revista Med. Instituto Mexicano Seguro Soc., 53: 294-301.

Chen, N., Y. Liu, Y. Cheng, L. Liu and Z. Yan et al., 2015. Technology resource, distribution and development characteristics of global influenza virus vaccine: A patent bibliometric analysis. PLoS ONE, 10: 1-19. DOI: 10.1371/journal.pone. 0136953

Cobo, M.J., A.G. Lopez-Herrera, E. Herrera-Viedma and F. Herrera, 2012. SciMAT: A new science mapping analysis software tool. J. Associat. Inform. Sci. Technol., 63; 1609-1630. DOI: 10.1002/asi.22688

Colledge, L. and R. Verlinde, 2014. SciVal Metrics Guidebook. 1st Edn., Elsevier, Netherlands, pp: 96.

Davenport, F.M., A.V. Hennessy, F.M. Brandon, R.G. Webster and C.D. Barrett Jr et al., 1964. Comparisons of serologic and febrile responses in humans to vaccination with influenza a viruses or their hemagglutinins. J. Lab. Clin. Med., 63: 5-13.

Francis, T., J.E. Salk, H.E. Pearson and P.N. Brown, 1945. Protective effect of vaccination against induced influenza A. J. Clin. Investigat., 24: 536-546.

Hou, J., 2014. Analysis on the research fronts of a H1N1 influenza by bibliometrics and information visualization. Zhonghua Liu Xing Bing Xue Za Zhi = Zhonghua Liuxingbingxue Zazhi, 35: 1284-1288.

Ilyichev, A., L. Karpenko, V. Gureyev and N. Mazov, 2016. Development of phage display technology: A bibliometric assessment. OnLine J. Biol. Sci., 16: 34-42. DOI: 10.3844/ojbsci.2016.34.42

Ilyicheva, T., I. Sobolev, I. Susloparov, O. Kurskaya and A. Durymanov et al., 2013. Monitoring of influenza viruses in Western Siberia in 2008-2012. Infection, Genet. Evolut., 20: 117-187. DOI: 10.1016/j.meegid.2013.08.025

Ilyicheva, T., I. Susloparov, A. Durymanov, A. Romanovskaya and K. Sharshov et al., 2011. Influenza A/H1N1pdm virus in Russian Asia in 20092010. Infection, Genet. Evolut., 11: 2107-2112. DOI: 10.1016/j.meegid.2011.05.002

Ilyicheva, T., M. Abdurashitov, A. Durymanov, I. Susloparov and N. Goncharova et al., 2016. Herd immunity and fatal cases of influenza among the population exposed to poultry and wild birds in Russian Asia in 2013-2014. J. Med. Virol., 88: 35-44. DOI: $10.1002 / \mathrm{jmv} .24301$

Ivanov, V.V., A.N. Libkind and V.A. Markusova, 2014. Publication activity and research cooperation between higher education institutions and the Russian Academy of Sciences. Her. Russ. Acad. Sci., 84: 28-34. DOI: 10.1134/s101933161401002x 
Jin, H. and K. Subbarao, 2014. Live attenuated influenza vaccine. Curr. Topics Microbiol. Immunol., 386: 181-204. DOI: $10.1007 / 82 \_20144410$

Krammer, F. and P. Palese, 2015. Advances in the development of influenza virus vaccines. Nat. Rev. Drug Discov. 14: 167-182. DOI: 10.1038/nrd4529

Mazov, N.A., V.N. Gureyev and M.I. Epov, 2017. Scientometric analysis of international scholarly output in borehole geophysics. Karotazhnik, 12: 65-86.
Svyatchenko, S.V., A.G. Durymanov, I.M. Susloparov, N.P. Kolosova and N.I. Goncharova et al., 2016. Severe and fatal influenza cases in Russia in 20142015. OnLine J. Biol. Sci., 16: 184-192.

DOI: $10.3844 /$ ojbsci.2016.184.192

WHO, 2009. WHO pulbic health research agenda for influenza. World Health Organization. 